\title{
AFM Integrated with SEM/FIB for Complete 3D Metrology Measurements
}

\author{
Aaron Lewis ${ }^{1}$, A. Komissar ${ }^{2}$, A. Ignatov², Oleg Fedoroyov², and E. Maayan ${ }^{2}$ \\ 1. The Department of Applied Physics, The Hebrew University of Jerusalem \\ 2. Nanonics Imaging Ltd, 19 Hartum St., BYNET Bldg, Jerusalem, Israel
}

Scanning electron microscopy (SEM) and ion beam milling (FIB) techniques are mature nanoscale measurement technologies, while Atomic Force Microscopy (AFM) is a developing technology with intense interest in the scientific community for basic research and development. We discuss the recent integration of these techniques into a single instrument enabled by technological innovation in AFM instrument design, and applications of this instrument for characterization of FIB milled trenches.

An AFM integrated into the SEM/FIB has specific design constraints that need to be met involving the AFM scanner, probe design, and feedback loop so that it geometrically fits into the chamber without interfering with the electron and ion beams. An ultraflat scanning stage was thus developed for precise sample motion that enable large ranges (85 microns or greater) in $\mathrm{x}, \mathrm{y}$, z. SPM probes that do not interfere with the electron beam were manufactured from fused silica in a cantilevered geometry (see Fig 1) where there is full visualization of the tip, without any loss of SPM functionality. These probes are combined with a tuning fork based feedback mechanism to maintain accurate tip-sample control; this mechanism does not involve lasers or optical beam deflection that interferes with the electron and ion beams.

The many benefits of this combined approach to three-dimensional nanoscale characterization are illustrated in the imaging of a FIB milled trench. Figure 2a shows an AFM image profiling a 25- $\mu \mathrm{m}$-deep trench milled in silicon with FIB imaged with the large $\mathrm{Z}$ range of the system. In this measurement, the FIB beam was used to mill this feature, and it was followed immediately by AFM imaging without having to remove the specimen from the FIB chamber and search in the AFM. Furthermore, the true depth of the trench was measured by the AFM in a crosssectional profile (Figure $2 \mathrm{~b}$ ) while a 3D reconstruction revealed the geometry of the sidewall (Figure 2c). Sidewall imaging with such long and exposed tips now are feasible with this instrument. The red arrow in this image clearly shows a Pt decoration on this structure, and the white arrow shows an undercut. Additionally, the ability of AFM to quickly and easily follow FIB milling with high $\mathrm{Z}$ resolution provides a straightforward and convenient method to check on FIB milling results. This capability is demonstrated in Figure 3 where several FIB-milled features in Si were monitored during the milling process. The grayscale SEM image in Figure 3a can easily locate these features and measure their widths but cannot provide direct information on their depths. The AFM delivers a 3D reconstruction (Figure 3b), and a cross sectional profile that together provide a true topographic map of specimen features. These depth profiles demonstrate the linearity of the etching process with time.

This instrument incorporates important innovations in the design and technology of atomic force microscopy enabling its integration with SEM and FIB into a single powerful capability. 


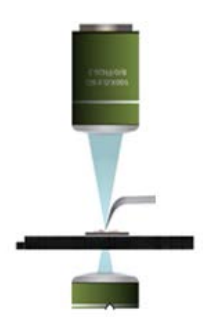

Figure. 1

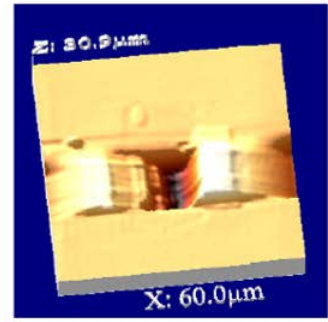

Figure 2a
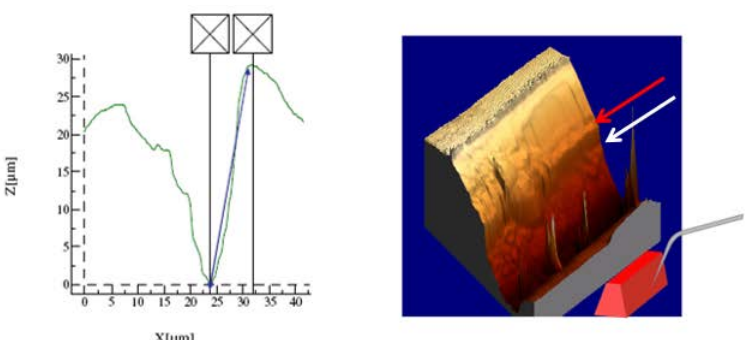

$2 b$

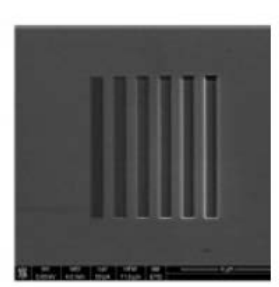

Figure 3a

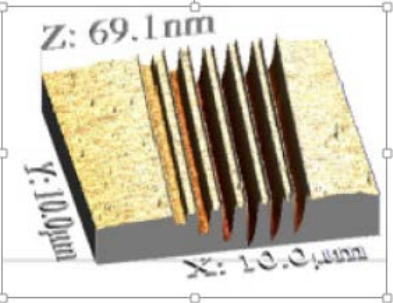

$3 b$

References

(1) Maier, S.; Kik, P.; Atwater, H. A.; Meltzer, S.; Harel, E.; Koel, B. E.; Requicha, A. g. Local detection of electromagnetic energy transport below the diffraction limit in metal nanoparticle plasmon waveguides. Nature Materials 2003, 2, 229.

(2) Prikulis, J.; K.Murty; Olin, H.; Kall, M. Large area topography analysis and near field Raman spectroscopy using bent fibre probes. Journal of Microscopy 2003, 210, 269-273.

(3) Raghu, D.; Hoffman, J. A.; Garnari, B.; Reeves, M. E. Near-field ablation threshold of cellular samples in the mid-infrared wavelength region. Appl Phys Lett 2012, 100, 203703.

(4) Dekhter, R.; Khachatryan, E.; Kokotov, Y.; Lewis, A. Investigating material and functional properties of static random access memories using cantilever glass multiple-wire force-sensing thermal probes. Appl Phys Lett 2000, 77, 4425.

(5) Ren, X.; Liu, A.; Zou, C.; wang, L.; Cai, Y.; Sun, F.; Guo, G.; Guo, G. Interference of surface plasmon polaritons from a "point" source. appl Phys Lett 2011, 98, 201113.

(6) Lewis, A.; Kheifetz, Y.; Shambrodt, E.; Radko, A.; Khatchatryan, E.; Sukenik, C. Fountain pen nanochemistry: atomic force control of chrome etching. Appl Phys Lett 1999, 75, 2689.

(7) Ternes, M.; Lutz, C. P.; Hirjibehedin, C. F.; Giessibl, F. J.; Heinrich, A. J. The force needed to move an atom on a surface. Science 2008, 319, 1066.

(8) Kohlgraf-Owens, D.; Sukhov, S.; Dogariu, A. Mapping the Mechanical Action of Light. Phys Review A 2011, 84, 011807.

(9) Little, A.; Hoffman, A.; Haegel, N. M. Optical attenuation coefficient in individual ZnO nanowires. Optics Express 2013, 21, 6321. 